\title{
Energy Efficient Algorithms for Wireless Sensor Network
}

\author{
Manikandan.K ${ }^{1}$, Kanmani. ${ }^{2}$, Munnira Sulthana. $M^{3}$ \\ PG Scholar, Sri Ramakrishna Institute of Technology, Coimbatore, India ${ }^{1}$ \\ Assistant Professor, Sri Ramakrishna Institute of Technology, Coimbatore, India ${ }^{2,3}$
}

\begin{abstract}
Now a day's big data has boomed as a hot topic. The data gathering, processing, and analysis of this big data are very challenging process. The sources of big data are widely spreader across the world. The audio, video, text and image files are the causes of the big data which is produced by $\mathrm{p} 2 \mathrm{p}$ application, real-time database processing, delivering contents, cloud storages, web contents, RFID and sensors. The main source of big data is the Wireless Sensor Networks. In WSNs we are using hundreds and thousands of sensor nodes. Each and every sensor nodes are monitoring the environment or the physical parameter and combining those data. The parameters are temperature, pressure, humanity etc. The energy of sensor nodes is the major role in Wireless Sensor Networks. Mostly the network failure happens because of security attack and lack of energy of sensor nodes. The life time of sensor nodes are limited. So the WSN should efficiently use the sensor nodes and the power or energy and also the throughput should be efficient. There are number of algorithms and protocols which were proposed to reduce the energy consumption. Here some important algorithms are studied for a better understanding of energy efficient algorithms in Wireless Sensor Networks.
\end{abstract}

Keywords: Wireless Sensor Network, Energy Efficient Algorithms and Protocols, sensor nodes, energy.

\section{INTRODUCTION}

Big data is nothing but "small data" but "bigger". By the IBM survey in 2012 the $90 \%$ of the data in the world was generated in last two years. Sure this will keep on increasing. So we all in the need of Big Data, which is used to analyze, gathering and processing the data. But the real sources of this Big Data are cloud storage, Web contents (social media), sensors etc. The big data is mostly being generated by the Industry, Government, and Academia.The most effective contributor of the big data is the sensors. Particularly the Wireless Sensor Networks plays a vital role. The wireless sensor networks are nothing but the collection of hundreds and thousands of small sensor nodes and small nodes monitor the physical or the environmental conditions. The WSN is designed of a large number of low-power sensor nodes. These nodes are widely spreader with limited computation and sensing operations. It has flexibility, low-cost fault tolerance characteristics. Because of these reasons the Wireless Sensor Networks are widely using in environment monitoring, industries, military, map, and vehicle technologies and so on. The parameters of sensor nodes which is used in the above areas are temperature, pressure, humanity etc.,

However the Wireless Sensor Networks has some limitations and limited computational capabilities. There are several types of challenges in the WSNs. They are,

* Time critical

* Sense or cover a huge geographical area

* The data may be faulty due to the environment which may be unreliable

* And the constant or the fixed routing path for communication is impossible

\# When the bandwidth for WSN is much lower

* Low buffer storage

Copyright to IJARCCE
These challenges must be overcome or handled before the sensor nodes of the Wireless Sensor Network gather the more complex data or information.

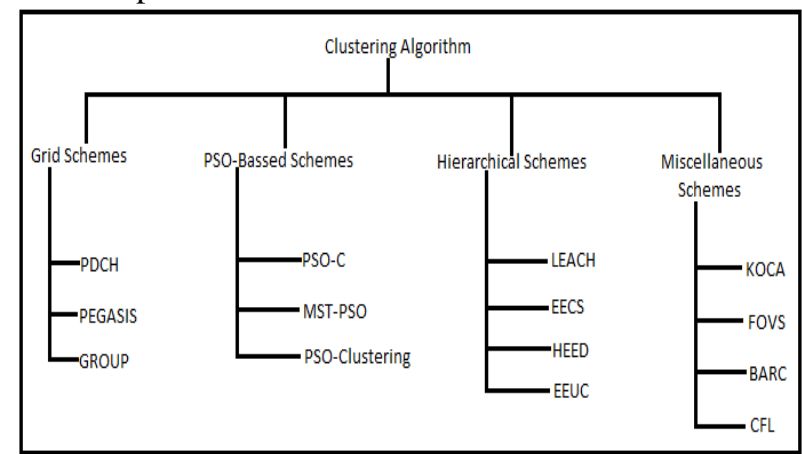

Fig. 1 Taxonomy of Clustering Algorithms

The main drawback of this WSN is the sensor nodes which are placed in densely distributed area in not capable or possible to recharge. As well as the fact that we cannot replace the batteries by another. These sensor nodes may die due to the lack of energy and it may leave a gap in the WSNs coverage area. In addition, these death nodes break the communication or network connectivity in the Wireless Sensor Network or any network. It is more difficult to recharge and also replace by another sensor node in their same Wireless Sensor Network. There is possibility to replace by another sensor node but the cost factor is more expensive.

Finally the solutions for the above drawbacks should be an optimal one. So in Wireless Sensor Network the results should be low cost, low latency, or quick response, fault tolerant and mainly the energy consumption should be minimum. The above Figure. 1 explains about the various types of clustering algorithms and it's family. It shoes that the numerous number of algorithms are used and each and 
every algorithms are has a different methodology. To achieve these common factors, there are more number of algorithms and protocols were invented and implemented. Those algorithms and routing protocols are focusing on reducing cost complexity, energy consumption, and coverage area of Wireless Sensor Network and each algorithms and protocols may suitable for every factor in an enough manner. But some of them focus only on a particular factor. In these algorithms and protocols, a few of them are selected and explained in the following section which is well suited for reducing the energy consumption of sensor nodes in Wireless Sensor Networks.

\section{Algorithms}

As mentioned earlier, there are a number of algorithms and protocols are there for reducing the energy consumption, low-latency, overcome the limited factors of each and every sensor nodes in sensor networks. From those algorithms few are the well suitable solution for the wireless sensor networks. Those are given below,

1) $L E A C H$ : This is the technique which is used to reduce the sum of square of communication range. The energy is evenly spreader to the all sensor nodes by this algorithm. So the network lifetime is increasing.

2) PEGASIS: This algorithm overcomes the drawback of LEACH algorithm. In LEACH, if the base station is far away from the cluster head means that cluster head have to spend more energy to transmit the data to BS. So that the corresponding cluster head will die soon. Thus this algorithm overcomes this issue.

3) KOCA: This algorithm is also the algorithm which is used to reduce energy consumption. The above algorithm is only focused on a cluster. But in KOCA, it handles the overlapping problem about two or more clusters. This is not more efficient but it handles more number of sensor nodes and clusters than other two algorithms.

The Algorithms which are listed above briefly explained below in the following section. Section A explains about the LEACH algorithm and its working mechanism. Section B describes about the PEGASIS algorithm and in section C KOCA or multi-hop clustering algorithm is explained.

\section{A. LOW-ENERGY ADAPTIVE CLUSTERING HIERARCHY}

By the name itself, Low-Energy Adaptive Clustering Algorithm or LEACH is self-organizing, and adaptive clustering protocol. LEACH is used a randomize technique to evenly distribute the energy and the evenly distribute the load of each and every node in the sensor network. In LEACH, the sensor nodes are assembling as a clusters called local clusters. That local cluster will have a cluster-head called as local base station. When the cluster head has chosen, all the other member nodes or noncluster-head node will send their data to the corresponding cluster-head in cluster. In conventional clustering algorithms, the sensor node which was selected as a cluster-head will die quickly due to the battery drain. So the network failure may happen easily, as well as quickly.

Thus the LEACH has a different method to save the lifetime of cluster head and also the overall network. The LEACH algorithm is using a technique called randomized rotation of the high-energy cluster-head selection. That means, this algorithm will choose the cluster-head periodically based on the sensor nodes having the highenergy. The cluster-head will be a local base station only for a particular time. Then after particular time, the cluster-head selection will repeat again based on the same energy parameter. In addition the $\mathrm{LEACH}$ will compress the data after gathered the data from all other member nodes. And it will send it to the base station. This process will reduce the energy consumption and also enhancing the lifetime of the system. When the sensor node is elected as a cluster-head in a cluster, that cluster head will broadcast the status to their member nodes or non-clusterhead nodes which belongs to their cluster in Wireless Sensor Network.

In LEACH based network, when the cluster is formed or organized, the cluster head will generate a schedule for their corresponding nodes of their own cluster. This for, each non-cluster-head comes to know their time slot for transmits the data to cluster head. So except that time slot all other radio components of the member nodes will be turned off. This key idea is greatly reduces the energy consumption during the idle time. Once the cluster-head has all the data from their member nodes, it will compress it and send it to the base station. Sometimes the base station is far away from the cluster-head. It will be a very high-energy transmission. So that cluster head sensor nodes' battery will drain soon. However, some node will be affected like this but when compared to other conventional algorithms; it is more efficient about energy consumption.

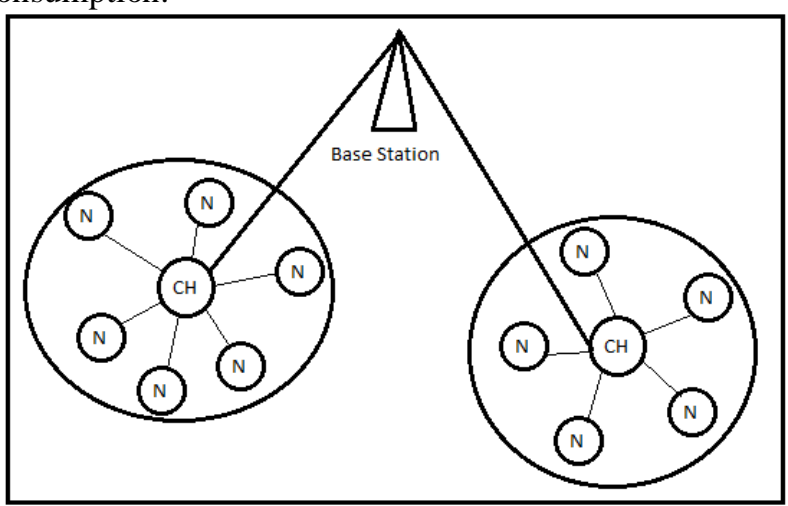

N- Member node,

CH- Cluster Head Node

Fig. 2 Simple LEACH Cluster Forming Approach

The functions and cluster formation technique is shown in Figure. 2, and it shows the duty of cluster head clearly. Where the $\mathrm{N}$ is denotes the node which is a member node or non-cluster head node. And the $\mathrm{CH}$ denotes the Cluster- Head node in the Wireless Sensor 
Network. As we mentioned earlier, the battery of cluster head nodes will drain soon because of the base station is placed far away from the cluster-head. So the cluster head nodes will not fixed or permanent in order to equalize the energy usage of all sensor nodes. For this reason, the node will select themselves as a cluster head from them in a particular time interval. The next cluster head will have chosen depends on the amount of energy left at the node currently. And the remaining functions will happen as like as mentioned above.

Thus the above advantages of the LEACH or LowEnergy Adaptive Clustering Hierarchy make that algorithm as a very famous algorithm for Wireless Sensor Networks.

\section{B. POWER EFFICIENT GATHERING IN SENSOR INFORMATION SYSTEM}

As mentioned earlier LEACH protocol forms a cluster, the cluster head will receives the data from all the nodes, compress that entire data, and send it to the base station finally. By randomized technique the energy consumption is reduced in LEACH algorithm. But still there is some drawbacks exist in LEACH algorithm. Such that consider, if the base station is far away from the cluster heads in the cluster. Those clusters heads will consume the more energy to transmit their data to the base station, so that, the cluster head will die soon due to the high transmission energy consumption. The network failure may occur because of this scenario at any time. Leach is more effective algorithm but still some nodes may die because of the illness of LEACH.

To address the issues of LEACH, PEGASIS protocol (Power-Efficient GAthering in Sensor Information System) proofs to be better. In PEGASIS the each nodes in the cluster will establish the communication transmission to the very closest node only. So the energy consumption will reduce while transmitting data to the base station.

In Wireless Sensor Network, the fusion plays a vital role to reduce the amount of data transmission from the sensor nodes and the base station. Data fusion is nothing but combines one or more packets, which is sensed from different sensor environment to produce a single packet. The LEACH protocol is a solution to reduce the energy consumption in data collection which forms clusters. Each cluster collects the data and fuses the data. Finally one cluster head will send it to the station about that result. These functions will about by randomized technique. But further improvement is possible by using the PEGASIS protocol. This improvement is obtained if each node communicates with their closest node in Wireless Sensor Network. In PEGASIS, the key idea is to construct a chain with each sensor node so that the node will transmit the data and receive the data to the closest neighbor nodes. Those data keep moving from a node to another node. Also these nodes are getting fused in each node and finally transmitted to the base station. So here, each and every node in the server network will spend their energy eventually for to do that work. Building a chain is similar to the travelling salesman problem.

The simple chain method is performed with a greedy approach. This approach is shown in Figure. 2 where each and every node is communicating with their closest neighbor nodes. And also it shows that the token passing method such that, if the node is center of two nodes, then that node will wait for that data from both to adjacent nodes. After collecting all the data from the both nodes, it will compress it and send it to the base station or the next nearest node. In PEGASIS, if any node is not in the communication range of each other, there is a possibility to use the multi-hop transmission paths. While collecting the data, no node should be left out; else the data of that node may be lost or missed for sequence data. The LEACH protocol is good enough to reach the base station within a communication range but PEGASIS overcome the drawback of LEACH such that if base station is far away from the cluster head. Finally the PEGASIS is more efficient in two ways compared to the LEACH protocol. That is,

* When transmitting the data of all sensor nodes to the cluster-head after fusing, PEGASIS takes much less distances because of the chain method.

And then the PEGASIS leader is receive the data from the more member nodes at a time. Then leader node will send that data to base station.

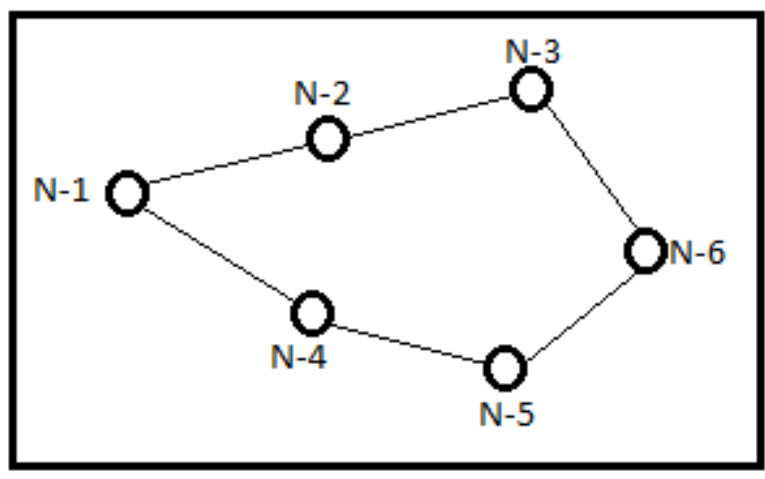

Fig. 3Simple Chain and Token passing method

Thus the PEGASIS is the extension of the LEACH in an efficient manner.

\section{K-HOP CLUSTERING ALGORITHM}

We know that the cluster is used for attain an energy efficiency and flexibility in wireless sensor networks. But in sensor networks we cannot assure that clusters won't be stable all the time. Some sensor nodes may interrupt into another cluster range. So collision will occur there. This function is called overlapping cluster problem. That means some sensor nodes belong to one cluster head will interact or interrupt into another cluster form. But this overlapping clustering is useful in some types of scenarios. Those are nothing but Inter-cluster routing, node localization, and time synchronization protocols. However, the overlapping cluster problem should be avoided in more scenarios due 
to the congestion. So here, k-hop clustering algorithm can be used for this type of scenario.

Normally the other clustering algorithms such as LEACH, PEGASIS will forms only the disjoint clusters. And also that will fulfill limited criteria. But by using the KOCA protocol, we can formulate a huge clustering and can handle energy efficiency parameter. In traditional clustering algorithm, each and every node will belong to only one cluster. But not in the case of multi-hop clustering or overlapping clustering algorithm. Here a single node may belong to more than one cluster. In the KOCA model there are 3 types of nodes in the each and every cluster. They are given below,

* Cluster Head $(\mathrm{CH})$

* Boundary Node (BN)

* Member or Normal node

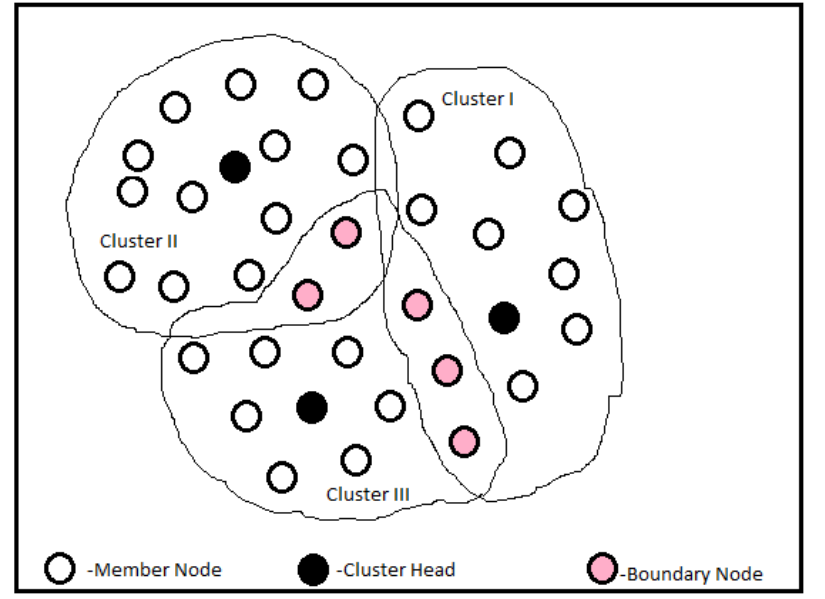

Fig. 4 Overlapping Clusters

The cluster head will choose by the particular probability. That cluster head will have the details of nearest clusters and also they know how to communicate with them and the boundary node is nothing but, it belongs to more than one cluster in the sensor network. The boundary node will act as a gateway. The figure. 3 shows the Member nodes, Cluster head nodes, and Boundary nodes. The cluster head selection is same as the LEACH and PEGASIS algorithms. There is no centralized control to select the cluster head and also like LEACH and PEGASIS, the KOCA sensor nodes also advertise which is the elected cluster head to all other non-cluster head or member nodes.

The KOCA algorithm is a challenging algorithm. The KOCA aims at connected overlapping clusters. That overlapping clusters will cover the whole sensor networks with the help of the boundary nodes. These boundary nodes will be at the overlapping area. The KOCA algorithm incurs the low overhead compared to other algorithms such as LEACH and PEGASIS. The key idea of the KOCA algorithm is that this algorithm considers the bidirectional links. Here for avoiding collision, we can use the multiple accesses with collision avoidance protocol and also the other MAC protocols like TDMA may be used for mac layer communication in overlapping clustering without the collision are occurring.

Thus the multi-hop clustering or the overlapping clustering is a better algorithm if the wireless sensor network has a very huge number of sensor nodes. The LEACH and PEGASIS algorithms are very optimal to reduce energy consumption but by considering one cluster. But in overlapping clustering algorithm the total entire sensor network has covered. The overlapping area is handled in a very effective manner. So this is better choice for a network which has huge number of sensor nodes.

\section{CONCLUSION}

There are numerous algorithms for reducing the energy consumption in Wireless Sensor Network. With the increase in data coming from wireless sensor nodes, there is a need to reduce energy consumption in wireless sensor nodes. Such energy efficient protocols were discussed here briefly. These algorithms have both their own advantages and certain limitations. According to the size of the sensor network, delay in the network, number of clusters a suitable algorithm may be implemented.

\section{REFERENCES}

[1] Daisuke Takaishi , Hiroki Nishiyama, Nei Kato, and Ryu Miura, "Towards Energy Efficient Big Data Gathering in Densely Distributed Sensor Networks", DOI 10.1109/TETC.2014.2318177, IEEE Transactions on Emerging Topics in Computing, 2014.

[2] M. Chen, T. Kwon, and Y. Choi, "Energy-efficient differentiated directeddiffusion (eddd) in wireless sensor networks," Computer Communications, vol. 29, no. 2, pp. 231-245, 2006.

[3] C. Intanagonwiwat, R. Govindan, and D. Estrin, "Directed Diffusion: ascalable and robust communication paradigm for sensor networks," inMobiCom'00 Proceedings of the 6th annual international conference on Mobile computing and networking, 2000.

[4] L. He, Z. Yang, J. Pan, L. Cai, J. Xu, and Y. Gu, "Evaluating servicedisciplines for on-demand mobile data collection in sensor networks,"vol. PP, no. 99, 2013.

[5] H. Nakayama, N. Ansari, A. Jamalipour, and N. Kato, "Faultresilientsensing in wireless sensor networks," Computer Communications, vol. 30, no. 11-12, pp. 2375-2384, Sep. 2007.

[6] S. Lindsey and C. Raghavendra, "PEGASIS: Power-efficient gatheringin sensor information systems," in IEEE Aerospace Conference, 2002.

[7] M. Youssef, A. Youssef, and M. Younis, "Overlapping multihop clusteringfor wireless sensor networks," IEEE Transactions on Parallel andDistributed Systems, vol. 20, no. 12, pp. 1844-1856, Dec. 2009.

[8] W. Heinzelman, A. Chandrakasan, and H. Balakrishnan, "Energyefficientcommunication protocol for wireless microsensor networks,"in Annual Hawaii International Conference on sytem Sciences, vol. 2,Jan. 2000.

[9] R. C. Shah, S. Roy, S. Jain, and W. Brunette, "Data MULEs: modellingand analysis of a three-tier architecture for sparse sensor networks," AdHoc Networks, vol. 1, no. 2-3, pp. 215 - 233, 2003.

[10] K. Baumgartner, S. Ferrari, and A. Rao, "Optimal control of an underwatersensor network for cooperative target tracking," IEEE Journal ofOceanic Engineering, vol. 34, no. 4, pp. 678-697, Oct. 2009.

[11] Vinay Kumar, Sanjeev Jain and sudarshanTiwari, "Energy Efficient Clustering Algorithms in Wireless Sensor Networks: A Survey", IJCSI International Journal of Computer Science Issues, sep 2011.

[12] S. Wen-Zhan, H. Renjie, X. Mingsen, B. Shirazi, and R. LaHusen,"Design and deployment of sensor network for real-time high-fidelity volcano monitoring," IEEE Transactions on Parallel and Distributed Systems, vol. 21, no. 11, pp. 1658-1674, Nov. 2010.

[13] C.-C. Lin, M.-J. Chiu, C.-C. Hsiao, R.-G. Lee, and Y.-S. Tsai, "Wirelesshealth care service system for elderly with dementia," IEEE Transactionson Information Technology in Biomedicine, vol. 10, no. 4, pp. 696-704, 2006. 
[14] C. Jiming, X. Weiqiang, H. Shibo, S. Youxian, P. Thulasiraman, and S. Xuemin, "Utility-based asynchronous flow control algorithm for wireless sensor networks," IEEE Journal on Selected Areas in Communications, vol. 28, no. 7, pp. 1116-1126, Sep. 2010.

[15] S. He, J. Chen, D. Yau, and Y. Sun, "Cross-Layer optimization of correlated data gathering in wireless sensor networks," in IEEE CommunicationsSociety Conference on Sensor Mesh and Ad Hoc Communications and Networks (SECON), Jun. 2010, pp. 1-9.

[16] CH.SITA KUMARI, CH.DEVI, B.KIRAN KUMAR, B.SANTOS KUMAR, K.JAGADISH CHANDRA, "A Modified Leach Algorithm to Enhancing the Performance with Improved Energy Efficiency for Wireless Sensor Network", International Journal of Application or Innovation in Engineering and Management, march 2013.

[17] S. Santhiya, A.Thamaraiselvi, "Survey on Energy Efficient Clustering Algorithms for wireless Sensor Network", International Journal of Latest Trends in Engineering and Technology, November 2013. 